\title{
Caso atípico de nefropatía por IgA: revisión práctica del protocolo diagnóstico en México
}

\author{
Macías Valadez-García Valeria*, Montoya-Padilla Claudia Judith*, Azúa-Díaz Gerardo Gilberto**
}

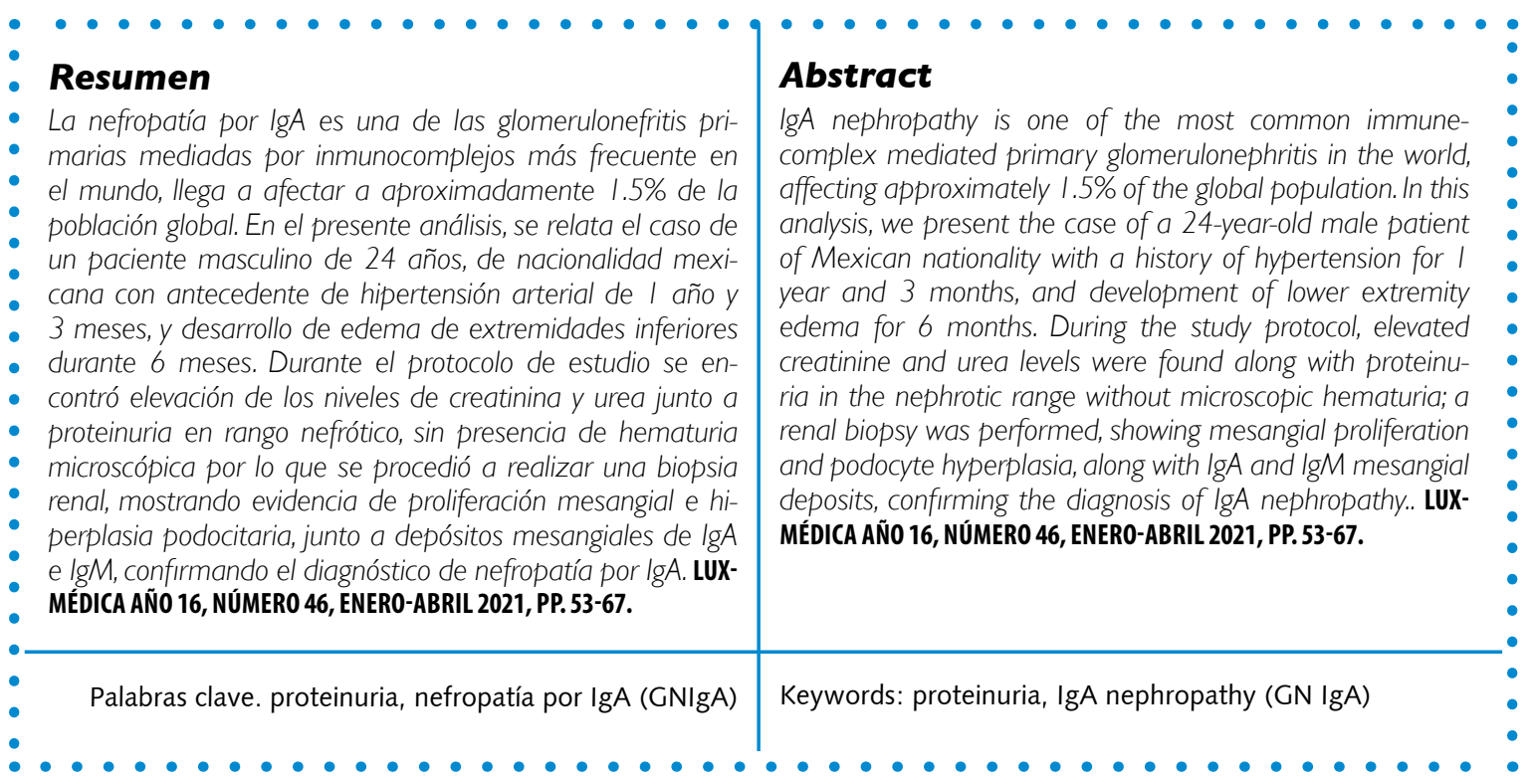

\section{Introducción}

La nefropatía o glomerulonefritis por IgA (GNIgA) es una de las glomerulonefritis primarias mediadas por inmunocomplejos más frecuente en el mundo, que afecta a $1.5 \%$ de la población global $_{i}{ }^{1}$ tiene mayor incidencia en varones entre la segunda y tercera década de la vida, ${ }^{2}$ con un promedio de $39.5 \pm 14$ años según los estudios anteriormente realizados..$^{3,4}$ En América Latina se ha observado que se presenta a menor edad, con un promedio de 30 años y mayor prevalencia en mujeres. La GNIgA representa una

Estudiante de la Carrera de Medicina del Centro de Ciencias de la Salud de la Universidad Autónoma de Aguascalientes. https://orcid.org/00000002-3932-2993. Correo electrónico valerim884@gmail.com

Estudiante de la Carrera de Medicina del Centro de Ciencias de la Salud de la Universidad Autónoma de Aguascalientes. https://orcid.org/00000003-1426-5184. Correo electrónico c.judith.m.p@gmail.com

** Profesor investigador de tiempo completo del Centro de Ciencias de la Salud de la Universidad Autónoma de Aguascalientes. https://orcid. org/0000-0002-8065-3487. Correo electrónico gerardo.azua@edu.uaa.mx

Fecha de recibido: 10 de septiembre 2020

Fecha de aceptación: 14 de diciembre de 2020

Correspondencia: Dr Gerardo Gilberto Azúa Díaz. Departamento de Medicina. Edificio 107 planta baja, Ciudad Universitaria. Universidad Autónoma de Aguascalientes. Avenida Universidad 930. Código postal 20131. Aguascalientes, Ags., México. Teléfono 4499108443. Correo electrónico gerardo.azua@edu.uaa.mx 
incidencia mayor en aquellos con descendencia europea y asiática, mientras que en Norteamérica se ha registrado una mayor incidencia en diabéticos con un $19.1 \% .{ }^{5}$

Un estudio realizado en América Latina, encontró que la nefritis lúpica (NL) (38.1\%) y la glomeruloesclerosis focal y segmentaria (GeFyS) (15.8\%) son las glomerulonefritis de mayor frecuencia, de acuerdo con las estadísticas de estudios realizados en Brasil, Colombia y México. ${ }^{5}$ En México se reporta una prevalencia de $7 \%$ de la GNIgA en adultos, después de la GeFyS (47\%) y la glomerulonefritis membranosa (GM) $(15 \%)$ en los patrones histológicos de las glomerulonefritis primarias. ${ }^{6}$ Otros estudios nacionales han reportado como diagnósticos histopatológicos más frecuentes la GM (27-34.8\%), ${ }^{6}$ el lupus eritematoso sistémico (LES) $(19.6 \%)$ y la GNIgA (10.9\%). ${ }^{7}$ En América Latina la incidencia de GNIgA es del $12 \%$ de las glomerulonefritis primarias. ${ }^{7}$

La GNIgA se trata de una glomerulonefritis de etiología desconocida; aunque en algunos estudios se ha demostrado ligada con 6q22-23 bajo un modelo dominante de penetrancia incompleta. ${ }^{8}$ La fisiopatología de esta patología es la generación de inmunoglobulinas IgA1, pobremente galactosiladas en su región bisagra donde predominan aminoácidos como la serina y treonina. En la GNIgA existe una galactosilación anormal de estos aminoácidos; en respuesta a estas alteraciones, el organismo genera la síntesis de IgG. Se generan complejos inmunes formados por IgG e IgA con glucosilación anormal, que se deposita en el mesangio generando la presencia de síndromes glomerulares variables que van desde anormalidades tubulares asintomáticas con hematuria microscópica, la presencia de hematuria macroscópica, síndrome nefrítico o glomerulonefritis rápidamente progresiva. Esta variabilidad de presentación es la que representa un reto clínico para la sospecha diagnóstica. En este caso, el paciente presentó un síndrome nefrótico incompleto, acompañado de hipertensión arterial.

Dentro de las manifestaciones clínicas, los pacientes con GNIgA, generalmente se presentan en una de las siguientes tres formas:

1 Del $40 \%$ al $50 \%$ se presentan con uno o más episodios de hematuria macroscópica, a menudo acompañado de infecciones de vías respiratorias superiores. Los pacientes pueden quejarse de dolor en el costado durante los episodios agudos, que generalmente reflejan el estiramiento de las cápsulas renales. La fiebre baja también puede estar presente. Estas características pueden imitar la infección del tracto urinario o la urolitiasis. La mayoría de los pacientes tienen sólo unos pocos episodios de hematuria macroscópica, y los episodios generalmente se repiten durante 2 a 3 años como máximo.

2 Del 30\% a 40\% tiene hematuria microscópica y generalmente proteinuria leve y se detectan de manera incidental en un exa- 
men de rutina o durante una evaluación diagnóstica de enfermedad renal crónica. En estos pacientes, la enfermedad es de duración incierta. La hematuria grave eventualmente ocurrirá en $20 \%$ a $25 \%$ de estos pacientes.

3 Menos del $10 \%$ presenta síndrome nefrótico o una glomerulonefritis aguda rápidamente progresiva, caracterizada por edema, hipertensión e insuficiencia renal, así como hematuria. En raras ocasiones, la GNIgA puede presentarse con hipertensión maligna. Por lo general, se presume que los pacientes tienen una enfermedad de larga evolución que no se detectó antes porque el paciente no tenía hematuria macroscópica ni se sometió a un análisis de orina de rutina. ${ }^{9}$

Anteriormente, la GNIgA se consideraba una enfermedad de curso benigno, pero debido a que puede evolucionar a enfermedad renal crónica terminal con necesidad de tratamiento sustitutivo hasta en un $50 \%$ de los casos en un lapso de 10 años, actualmente es considerado importante obtener un diagnóstico clínico e histológico de manera rápida y evaluar los diferentes factores que intervienen en la progresión de la enfermedad como son la presión arterial, sexo, edad, rango de proteinuria y porcentaje de fibrosis glomerular e intersticial en la biopsia. ${ }^{8}$

El objetivo de este trabajo es presentar el desarrollo clínico de la GNIgA en un paciente joven mexicano, cuya manifestación clínica inicial fue el desarrollo de hipertensión arterial secundaria, alteración en la función renal y proteinuria en rango nefrótico, sin presencia de hematuria.

\section{Presentación de caso clínico}

Acude paciente masculino de 24 años, con antecedente de hipertensión arterial de 1 año y 3 meses de evolución, manejada con telmisartán (40 mg vía oral cada 12 horas) y nifedipino (30 mg cada 12 horas), con respuesta regular y elevaciones persistentes de la presión arterial por la noche. Antecedente de obesidad grado 2 durante su adolescencia, trastorno depresivo mayor acompañado de distimia en los últimos 3 años, tratado con escitalopram $10 \mathrm{mg}$ por la mañana, aripiprazol $15 \mathrm{mg}$ por la noche y clonazepam $2.5 \mathrm{mg}$. Dislipidemia mixta de 2 años de evolución con manejo nutricional de forma intermitente. El motivo de consulta es la presencia de cefalea frontal pulsátil intermitente, edema hasta 1/3 medio de ambas tibias de 6 meses de evolución con godete positivo. Muestra exámenes de laboratorio con elevación de urea y creatinina a lo largo de los últimos 6 meses con cifra máxima alcanzada de creatinina de $2.5 \mathrm{mg}$ y urea de $90 \mathrm{mg} / \mathrm{dl}$. Negó la presencia de hematuria macroscópica durante el tiempo de evolución. Los hallazgos a la exploración física sin relevancia, sólo encontrándose un índice de masa corporal (IMC) de $36.71 \mathrm{~kg} / \mathrm{m} 2$, y una circunferencia de cintura de $130 \mathrm{~cm}$.

Se inicia protocolo de estudio obteniendo lo siguientes resultados de laboratorio (tabla 1). 


\section{Tabla I}

\section{Resultados de estudio de laboratorio (enero 2020)}

\begin{tabular}{|c|c|c|c|}
\hline Hemoglobina & $16.8 \mathrm{~g} / \mathrm{dl}$ & Creatinina & $1.6 \mathrm{mg} / \mathrm{dl}$ \\
\hline Leucocitos & $6.69 \mathrm{miles} / \mathrm{u}$ & Ácido úrico & $6.9 \mathrm{mg} / \mathrm{dl}$ \\
\hline Plaquetas & 312 miles/u & Fósforo & $4.1 \mathrm{mg} / \mathrm{dl}$ \\
\hline TP-TPT & $13.86 \mathrm{seg}-37.1 \mathrm{seg}$ & Calcio & $9.2 \mathrm{mg} / \mathrm{dl}$ \\
\hline Glucosa & $88 \mathrm{mg} / \mathrm{dl}$ & Potasio & $4.6 \mathrm{mmol} / \mathrm{L}$ \\
\hline Urea & $51 \mathrm{mg} / \mathrm{dl}$ & Sodio & $140 \mathrm{mmol} / \mathrm{L}$ \\
\hline $\begin{array}{l}\text { Complemento } \\
(\mathrm{C} 3, \mathrm{C} 4)\end{array}$ & Normal & $\begin{array}{c}\text { Panel viral (AgsHB, } \\
\text { HIV, VHC) }\end{array}$ & Negativo \\
\hline \multicolumn{4}{|c|}{ Examen General de Orina } \\
\hline Densidad & 1.0101 & Eritrocitos $\mathrm{x}$ campo & 2 \\
\hline $\mathrm{pH}$ & 6 & Leucos $x$ campo & 1 \\
\hline Proteínas & + & Urocultivo & - \\
\hline Colesterol & $201 \mathrm{mg} / \mathrm{dl}$ & Índice Aterogénico & 6.93 \\
\hline HDL & $29 \mathrm{mg} / \mathrm{dl}$ & Proteinuria & $4.66 \mathrm{gr} / 24 \mathrm{hrs}$ \\
\hline LDL & $83 \mathrm{mg} / \mathrm{dL}$ & Volumen de orina & $2600 \mathrm{ml}$ \\
\hline Triglicéridos & $445 \mathrm{mg} / \mathrm{dL}$ & Depuración de creatinina & $76.45 \mathrm{l} / \mathrm{min} / 1.7 \mathrm{~m}^{2}$ \\
\hline
\end{tabular}

Se calculó el filtrado glomerular (FG) a través de CKD-EPI con un resultado de $59.4 \mathrm{ml} / \mathrm{min} / 1.73 \mathrm{~m}$, compatible con el diagnóstico de enfermedad renal crónica grado 3 (ERCG3), con proteinuria en rango nefrótico y se corrobora la persistencia de dislipidemia mixta. Se obtiene un índice de masa corporal (IMC) de $36.71 \mathrm{~kg} / \mathrm{m} 2$ asociada a obesidad grado 3 .

En el estudio del ultrasonido renal realizado con transductor convexo multifrecuencia, se encontró el riñón derecho de $107 \times 42.2 \times 62.2 \mathrm{~mm}$ y de $107 \times 47.6$ x $52.9 \mathrm{~mm}$ el riñón izquierdo (imagen 1), ambos con localización normal, y sin presencia de signos de litiasis, tumoración, adelgazamiento del parénquima renal ni hidronefrosis en ninguno de los dos riñones. La relación córtico-medular fue de médula de $18.9 \mathrm{~mm}$ y corteza de $9.6 \mathrm{~mm}$ en riñón derecho y en el izquierdo fue de médula de $15.4 \mathrm{~mm}$ y corteza de $8.7 \mathrm{~mm}$ (imagen 2). Los uréteres no presentaban dilatación. En conclusión, se tiene la impresión de que ambos riñones tienen un margen ligeramente lobulado con incremento de ecogenicidad, la visibilidad de la pirámide se encontró disminuida. 


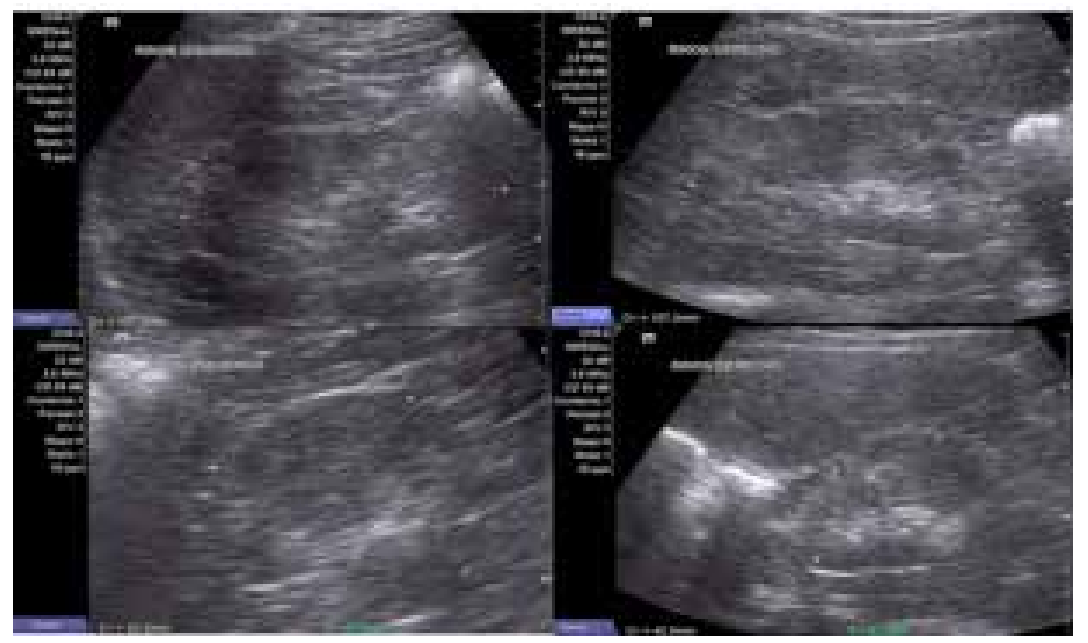

Imagen 1. Identificación de las medidas ultrasonográficas de cada uno de los riñones. La longitud renal máxima de ambos riñones es de $107 \mathrm{~cm}$.

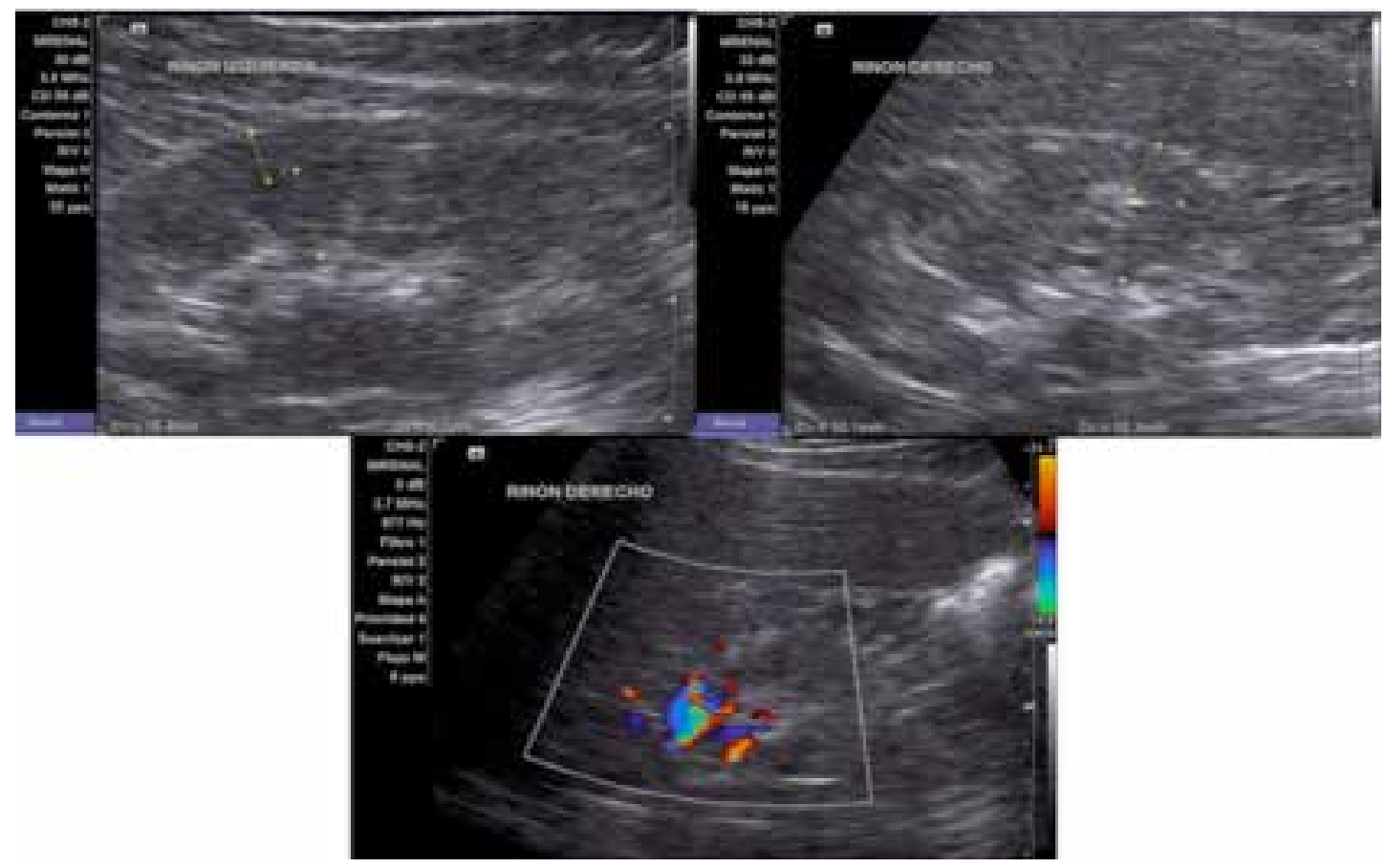

Imagen 2. Relación córtico-medular de cada uno de los riñones.

Doppler sin presencia de datos de obstrucción.

Con los parámetros anteriores se decide la toma de una biopsia renal percutánea, dos semanas posteriores a la obtención de laboratorios. Se describe el siguiente informe anatomopatológico: Macroscópicamente se identificó arteriolonefroesclerosis, proliferación mesangial, hiperplasia de los podocitos (glomerulomegalia), 
atrofia tubular del $30 \%$ y en las arteriolas preglomerulares se presenta hialinosis e hiperplasia de la media. Encontrándose en la inmunofluorescencia, un patrón mesangial de IgM e IgA (imagen 3).
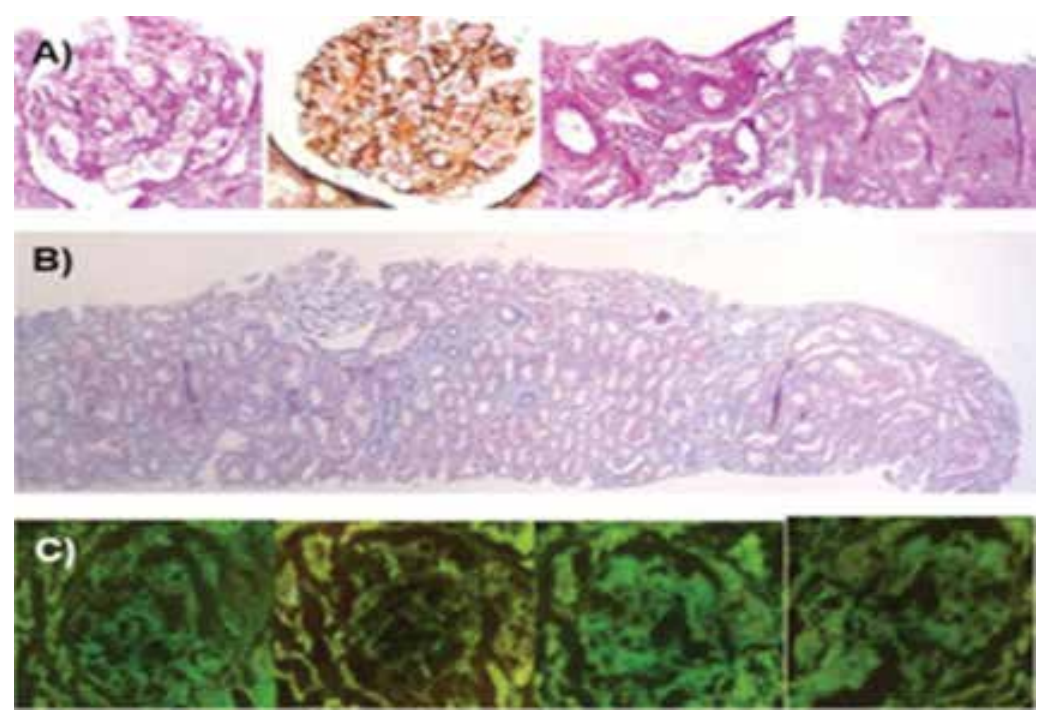

Imagen 3. A) Se observa glomerulomegalia e hiperplasia de podocitos y proliferación mesangial. B) Se muestran túbulos cilíndricos hialinos con atrofia y proliferación mesangial. C) Inmunofluorescencia en la cual se identifican depósitos mesangiales de IgA e IgM.

\section{Discusión}

En el paciente la GNIgA debutó a una corta edad y además fue acompañada de factores de mal pronóstico para la función renal de acuerdo con las pruebas clínicas, de laboratorio e inmunohistológicas. Además, la presentación clínica de este caso fue diferente, debido a que debutó con hipertensión arterial y proteinuria en rango nefrótico, pero sin hematuria.

La GNIgA se caracteriza por el depósito mesangial difuso de complejos IgA-IgG en los glomérulos, clínicamente suele presentar episodios recurrentes de hematuria macroscópica acompañada por infecciones del tracto respiratorio alto, o hematuria microscópica con o sin proteinuria, falla renal aguda que evoluciona a insuficiencia renal crónica y síndrome nefrótico. La GNIgA puede ocurrir en un contexto sistémico manifestándose como púrpura de Henoch-
Schonlein o de manera glomerular primaria, también conocida como enfermedad de Berger. ${ }^{1}$

Los estudios realizados en México reportan que la edad de presentación de la GNIgA en promedio es de $36 \pm 13$ años; existen distintas hipótesis que intentan explicar este hallazgo, algunos señalan las diferencias genéticas entre la población, la exposición ambiental a antígenos o agentes que pudieran inducir la enfermedad, como propone la hipótesis de la higiene. ${ }^{10}$

En México, dentro de las comorbilidades más frecuentes asociadas a GNIgA, son la hipertensión arterial (HTA) (24\%) y la diabetes mellitus tipo 2 (DM2) (4\%), además de la existencia de antecedentes familiares de ERC (10\%), ${ }^{10}$ que en este caso no se pudo consignar. Se conocen como indicadores clínicos y laboratoriales de mal pronóstico, al momento de la presentación 
de la enfermedad, a la edad avanzada, el sexo masculino, síndrome nefrótico, hematuria, presión arterial elevada, síntomas sistémicos, antecedentes familiares, tipo de HLA y elevación de creatinina. ${ }^{9}$ Nuestro paciente presenta presión arterial elevada, sexo masculino, la elevación de la creatinina y el síndrome nefrótico.

En algunos estudios se ha encontrado asociación entre hipertensión arterial y GNIgA posicionando a la hipertensión arterial como un indicador de mal pronóstico como anteriormente mencionamos, ya que se asocia a un mayor riesgo de enfermedad renal progresiva, además de que el riesgo de muerte es aproximadamente $40 \%$ mayor que en aquellos pacientes que tienen nefropatía por IgA sin hipertensión arterial. $^{9}$

Dentro de los indicadores de laboratorio que se consideran de mal pronóstico son: la creatinina sérica elevada, la presencia y magnitud de proteinuria, la microalbuminuria, la activación del complemento de C3, aumento de la excreción de IgG e incremento del colesterol sérico. La presencia de estos indicadores nos predice el riesgo de empeoramiento de la función renal, definida como la disminución del $50 \%$ en la tasa de filtrado glomerular o el desarrollo de enfermedad renal en etapa terminal, llegándose a presentar dentro de cinco a diez años del desarrollo de la enfermedad. ${ }^{9}$

En el paciente descrito ya existe lesión histológica establecida como hiperplasia de podocitos (glomerulomegalia), proliferación mesangial, arteriolonefroesclerosis a nivel vascular intrarrenal y atrofia tubular del $30 \%$, esto en conjunto genera alteración en la función renal, con elevación de creatinina y urea. En este punto es importante señalar que el predictor laboratorial más contundente de la presencia de lesiones histológicas como la glomeruloesclerosis o glomerulomegalia dentro de una biopsia renal son: 1) una tasa de filtrado glomerular $<30 \mathrm{ml} / \min$ por 1,73 $\mathrm{m}^{2}$, con elevación de creatinina y urea sérica,
2) presentación con síndrome nefrótico o proteinuria rango nefrótico y 3) lesión renal aguda. ${ }^{11}$ En el caso de nuestro paciente, el no encontrar glomeruloesclerosis dentro de la biopsia, actúa como factor protector. Además, la misma hipertensión establece un mecanismo de compensación hemodinámica con hiperfiltración glomerular por parte del resto de nefronas menos afectadas, generando un mejor patrón de filtrado glomerular con disminución de creatinina y urea sérica. Este hallazgo puede generar incertidumbre de si la función renal será capaz de mejorar con el paso del tiempo, ya que el no presentarlo se asocia a un mejor pronóstico funcional. Pero es importante señalar que el enfoque del tratamiento inicial y para los próximos años se enfoca en evitar un excesivo uso de hiperfiltración glomerular compensatoria, ya que ésta, a mediano y largo plazo, se asocia a la presencia de glomeruloesclerosis manifestándose con empeoramiento de la función renal. Este punto se correlaciona con el hallazgo de que el grado de función renal mejoró al inicio del protocolo de estudio, debido a que la cifra de creatinina sérica disminuyó a pesar de la ausencia del control de la presión arterial en los meses previos.

Habitualmente los pacientes con GNIgA que presentan poca o ninguna proteinuria (menos de 1000 mg/día) generalmente tienen un bajo riesgo de progresión. Entre los pacientes que desarrollan proteinuria manifiesta o una concentración elevada de creatinina sérica, la progresión a la enfermedad renal en etapa terminal es aproximadamente del $15 \%$ al $25 \%$ a los 10 años y del $20 \%$ al $30 \%$ a los 20 años. El promedio de proteinuria en $24 \mathrm{~h}$ para los pacientes con síndrome nefrótico, por GNIgA, es de $10.6 \pm 6 \mathrm{~g} /$ día en la población mexicana. ${ }^{11,12} \mathrm{Al}$ realizarle los exámenes de laboratorio al paciente, resultó con $4.66 \mathrm{gr} / 24$ hrs de proteinuria, lo cual explica el edema blando y caliente con fóvea de miembros inferiores. 
La presencia de síndrome nefrótico en la GNIgA es poco frecuente (3-7\%), y cuando se presenta, suele ser en estadios avanzados de la enfermedad, en promedio a 5 y 7 años; sin embargo, existen casos en los que se presenta al inicio, esto es indicación de realización de biopsia renal en $52 \%$ de los casos en adultos. ${ }^{13}$ En la biopsia renal, los hallazgos asociados a proteinuria en rango nefrótico en un paciente con GNIgA suelen ser la enfermedad de cambios mínimos (afección podocitaria) y proliferación mesangial. ${ }^{10}$ Para el paciente del caso, el hallazgo fue positivo para la proliferación mesangial y la hiperplasia podocitaria.

La obesidad y el sobrepeso son actualmente problemas graves en el área de la salud con tendencia creciente. Hay relativamente pocos estudios que abordan el tema de la obesidad en relación con la GNIgA, pero se ha demostrado que los pacientes obesos presentan más frecuentemente glomerulomegalia y glomeruloesclerosis secundaria, cuya traducción clínica es en forma de proteinuria de grado variable, sin consolidarse en un síndrome nefrótico completo. En el caso de la GNIgA se ha observado que la obesidad se asocia a una mayor velocidad de pérdida de función renal, en donde un IMC > $25 \mathrm{~kg} / \mathrm{m}^{2}$ es un buen predictor de aumento de la creatini- na. ${ }^{10,14}$ Para el paciente del caso clínico la obesidad y un IMC alto, son factores asociados al grado de proteinuria nefrótica, siendo una meta de tratamiento mejorar el grado de obesidad para disminuir el rango de proteinuria. La hipercolesterolemia también es considerada dentro del espectro de dislipidemia asociada a obesidad, en específico, en este paciente probablemente sea una afección previa al desarrollo de GNIgA, pero también actúa disminuyendo el pronóstico funcional renal, por lo que es motivo de manejo.

Dentro de los hallazgos patológicos en la biopsia renal, se consideran de mal pronóstico la presencia de arteriolonefroesclerosis, atrofia tubular, fibrosis intersticial, engrosamiento de la pared capilar glomerular y los depósitos de IgA. En el paciente se presenta arteriolonefroesclerosis, hialinosis arteriolar y fibrosis tubulointersticial que ha generado una atrofia tubular del $30 \%$. De acuerdo con las características inmunopatológicas, los hallazgos clínicos y los recabados en el laboratorio, el paciente se cataloga en riesgo severo según la clasificación de Tanaka S. y cols. con un score entre 15 y 22 puntos teniendo una incidencia entre 25 y $50 \%$ de evolucionar a ERC terminal a los 5 años $^{15}$ (tabla 2).

\section{Tabla 2}

\section{Score de Tanaka para estimar a cinco años el riesgo de evolucionar a una enfermedad renal crónica terminal}

\begin{tabular}{|c|c|c|c|c|c|c|}
\hline \multicolumn{2}{|c|}{ Riesgo para el desarrollo de ESRD } & \multicolumn{5}{|c|}{ Predicción de riesgo a los 5 años } \\
\hline Variables & Puntaje & $\begin{array}{c}\text { Puntaje del } \\
\text { paciente }\end{array}$ & $\begin{array}{c}\text { Puntaje de } \\
\text { riesgo total }\end{array}$ & $\begin{array}{c}\text { Riesgo absoluto } \\
\text { previsto a 5 años }\end{array}$ & $\begin{array}{c}\text { Puntaje de } \\
\text { riesgo total }\end{array}$ & $\begin{array}{c}\text { Riesgo abso- } \\
\text { luto previsto } \\
\text { a 5 años }\end{array}$ \\
\hline \multicolumn{2}{|c|}{ Excreción urinaria de proteínas } & 0 & 0.07 & 21 & 33.10 \\
\hline$<0.5 \mathrm{~g} / 24 \mathrm{hr}$ & 0 puntos & $(4.66 \mathrm{gr} / 24 \mathrm{hrs})$ & 1 & 0.10 & 22 & 41.90 \\
\hline $0.5-1.0 \mathrm{~g} / 24 \mathrm{hr}$ & 4 puntos & & 2 & 0.12 & 23 & 51.90 \\
\hline $1.0-3.5 \mathrm{~g} / 24 \mathrm{hr}$ & 6 puntos & 9 puntos & 3 & 0.18 & 24 & 62.80 \\
\hline$\geq 3.5 \mathrm{~g} / 24 \mathrm{hr}$ & 9 puntos & & 4 & 0.24 & 25 & 73.70 \\
\hline
\end{tabular}




\begin{tabular}{|c|c|c|c|c|c|c|}
\hline \multicolumn{3}{|c|}{ Estimación de tasa de filtrado glomerular } & 5 & 0.33 & 26 & 83.50 \\
\hline $\begin{array}{c}\geq 60 \mathrm{ml} / \\
\mathrm{min} / 1.73 \mathrm{~m}^{2}\end{array}$ & 0 puntos & \multirow{4}{*}{$\begin{array}{c}(59.4 \mathrm{ml} / \\
\left.\mathrm{min} / 1.73 \mathrm{~m}^{2}\right) \\
1 \text { Punto }\end{array}$} & 6 & 0.45 & 27 & 91.20 \\
\hline $\begin{array}{c}30-59 \mathrm{ml} / \\
\mathrm{min} / 1.73 \mathrm{~m}^{2}\end{array}$ & 1 punto & & 7 & 0.60 & 28 & 96.20 \\
\hline $\begin{array}{c}15-29 \mathrm{ml} / \\
\mathrm{min} / 1.73 \mathrm{~m}^{2}\end{array}$ & 4 puntos & & 8 & 0.81 & 29 & 98.80 \\
\hline $\begin{array}{c}<15 \mathrm{Ml} / \\
\mathrm{min} / 1.73 \mathrm{~m}^{2}\end{array}$ & 9 puntos & & 9 & 1.09 & 30 & 99.70 \\
\hline \multicolumn{3}{|c|}{ Medidas patológicas (Clasificación de Oxford) } & 10 & 1.47 & $\geq 31$ & 100.00 \\
\hline \multicolumn{3}{|c|}{ 1.- Hipercelularidad mesangial } & 11 & 1.98 & \multirow{10}{*}{\multicolumn{2}{|c|}{$\begin{array}{l}\text { El paciente se cataloga } \\
\text { en riesgo severo según } \\
\text { la clasificación de Tanaka } \\
\text { con un puntuación entre } \\
15 \text { y } 22 \text { puntos y con } \\
\text { probabilidad entre } 25 \text { y } \\
50 \% \text { de evolucionar a ERC } \\
\text { terminal a los } 5 \text { años. }\end{array}$}} \\
\hline $\begin{array}{c}\text { MO (s } 0.5 \mathrm{del} \\
\text { glomérulo) }\end{array}$ & 0 puntos & \multirow{2}{*}{2 Puntos } & 12 & 2.66 & & \\
\hline $\begin{array}{l}\text { M1 (> } 0.5 \text { del } \\
\text { glomérulo) }\end{array}$ & 2 puntos & & 13 & 3.58 & & \\
\hline \multicolumn{3}{|c|}{ 2.- Glomeruloesclerosis segmentaria } & 14 & 4.80 & & \\
\hline SO (ausente) & 0 puntos & \multirow{2}{*}{0 Puntos } & 15 & 6.42 & & \\
\hline S1 (presente) & 4 puntos & & 16 & 8.57 & & \\
\hline \multicolumn{3}{|c|}{ 3.- Fibrosis intersticial / Atrofia tubular } & 17 & 11.40 & & \\
\hline $\mathrm{TO}(\leq 25 \%)$ & 0 puntos & \multirow{3}{*}{6 Puntos } & 18 & 15.10 & & \\
\hline T1 $(26 \%-50 \%)$ & 6 puntos & & 19 & 19.80 & & \\
\hline $\mathrm{T} 2(>50 \%)$ & 10 puntos & & 20 & 25.70 & & \\
\hline
\end{tabular}

A pesar de los factores de mal pronóstico que presentaba el paciente al momento de iniciar el protocolo de estudio, se encontró una disminución de creatinina de 2.1 $\mathrm{mg} / \mathrm{dl}$ a $1.6 \mathrm{mg} / \mathrm{dl}$. La cifra de creatinina de 2.1 reportada dos meses previos al inicio del protocolo de estudio probablemente esté relacionado con la administración de medicamentos bloqueadores de la angiotensina II. La meta de manejo será evitar la hiperfiltración glomerular compensatoria para mejorar el pronóstico funcional renal y disminuir la proteinuria presente.

Normalmente el riñón izquierdo suele ser discretamente mayor que el derecho, además de que se encuentra posicionada más en lo alto. Un riñón sano suele medir de 10 a $12 \mathrm{~cm}$ : cuando se encuentra una diferencia de longitud entre ambos riñones mayor de $1.5 \mathrm{~cm}$ se considera significativa.
De manera normal o habitual, al explorar por ultrasonografía, se aprecia la anatomía renal constituida de una cápsula, el parénquima y el seno, de los cuales todos se observan hiperecogénicos, a excepción del parénquima que aparecerá hipoecogénico, en algunas ocasiones las pirámides medulares pueden observarse de un color más hipoecoico en comparación con el parénquima. En varios estudios se ha concluido que los pacientes con enfermedad renal crónica presentan riñones disminuidos de tamaño, desestructurados o con parénquima adelgazado. ${ }^{16}$

En el estudio del presente caso se encontró que las medidas del riñón se encuentran dentro del parámetro normal, con una longitud renal máxima de ambos riñones de $107 \mathrm{~mm}$. Debido a que el paciente es hipertenso, la ecografía nos ayu- 
dó a descartar una hipertensión secundaria como lo es por una estenosis de la arteria renal, la cual se deja de lado como etiología de hipertensión en nuestro paciente, ya que no se reporta asimetría renal, indicando que pudiera ser propia de la nefropatía por IgA. El grosor cortical normal mide alrededor de $1.1+-0.9 \mathrm{~cm}$, estándares a los cuales se apegó el sujeto de estudio. Ésta suele tener una relación de 1:1 con respecto a la médula renal, identificamos que ésta se encuentra disminuida en el riñón izquierdo, lo cual se puede correlacionar con el hallazgo reportado en la disminución de la visibilidad de la pirámide renal. Se concluyó que ambos riñones tienen un margen ligeramente lobulado con incremento de ecogenicidad, este dato se correlaciona con los datos reportados en la biopsia renal sobre la presencia de necrosis tubular, debido a que nuestro caso se trata de una persona joven, la hiperecogenicidad incrementada, no se puede justificar por la presencia de fibrosis parenquimatosa, que se suele presentar en personas de mayor edad. En los resultados del reporte ultrasonográfico no se visualizó hidronefrosis ya que, de presentarse, se encontraba visible el sistema pielocalicial, además de que en el doppler no se encontró la presencia de obstrucción urinaria (imagen 3 ).

Estudios anteriormente realizados han encontrado que la administración de bloqueadores de angiotensina II tienen mayores beneficios significativos que con la administración de otros antihipertensivos.
Dichos beneficios son: una tasa más lenta de pérdida del aclaramiento de creatinina y una mayor tasa de remisión de proteinuria, beneficios que se comienzan a observar cuando la presión arterial llega a cifras consideradas como normales. ${ }^{17,18} \mathrm{Un}$ punto importante es que la inhibición de la angiotensina no parece tener beneficio en aquellos pacientes no proteinúricos o en aquellos con bajos niveles de excreción de proteínas. $^{19}$

Otro punto para definir, de acuerdo con las características inmunológicas de la enfermedad, es la decisión de iniciar inmunosupresión. Para este paciente en particular, la decisión de iniciar inmunosupresión fue a través del sistema de clasificación de Tanaka. El paciente presenta un puntaje aproximado de 18 con un riesgo absoluto 15 veces mayor de progresar a ERC terminal en 5 años, clasificándose como de riesgo severo.

En KDIGO 2020 se hace mención de que la GNIgA sólo puede ser diagnosticada mediante una biopsia renal, puesto que actualmente no existen pruebas diagnósticas con biomarcadores serológicos o urinarias aprobadas. Posteriormente a ello, se debe proceder a la revisión de la muestra histológica guiándonos con base en la clasificación MEST-C de Oxford (ver tabla 3) y además se debe indagar acerca de la posibilidad de que la GNIgA fuera resultado secundario a alguna otra patología primaria de alguna etiología viral, autoinmune, cirrosis, enfermedad inflamatoria intestinal, entre otras.

\section{Tabla 3}

\section{Clasificación MEST-C de Oxford para GNIgA}

\begin{tabular}{|c|l|l|}
\hline MEST & \multicolumn{1}{|c|}{ DESCRIPCIÓN } & \multicolumn{1}{c|}{ PUNTAJE } \\
\hline$M$ & Hipercelularidad mesangial & $\begin{array}{l}\text { MO: }<50 \% \text { glomérulos } \\
\text { M1: }>50 \% \text { glomérulos }\end{array}$ \\
\hline E & Hipercelularidad endocapilar & $\begin{array}{l}\text { E0: Ausente } \\
\text { E1: Presente }\end{array}$ \\
\hline$S$ & Glomeruloesclerosis segmental & $\begin{array}{l}\text { S0: Ausente } \\
\text { S1: Presente }\end{array}$ \\
\hline
\end{tabular}




\begin{tabular}{|c|c|c|}
\hline $\mathrm{T}$ & Atrofia tubular & $\begin{array}{l}\text { T0: Ausente o }<25 \% \text { túbulos } \\
\text { T1: } 26-50 \% \text { túbulos } \\
\text { T2: }>50 \% \text { túbulos }\end{array}$ \\
\hline C & Semilunas fibrocelulares & $\begin{array}{l}\text { C0: Ausente } \\
\text { C1: } 1-24 \% \text { glomérulos } \\
\text { C2: }>25 \%\end{array}$ \\
\hline
\end{tabular}

Actualmente, se encuentra limitado la estadificación y tratamiento de pacientes de nefropatía IgA, aunque se trate de una enfermedad con ciertos criterios clínicos e histológicos, en cada individuo se presentan de manera particular, por lo que se dificulta su uso para estadificar la gravedad del paciente, considerando además la raza/ etnia que influye en el comportamiento de la enfermedad, ocasionando que el intento por estadificar la gravedad de los pacientes sea limitado en la práctica clínica.

Mediante los valores de eGFR y proteinuria de cada paciente, se puede valorar el pronóstico utilizando The International IgAN Prediction Tool se lograron validar el uso de 2 modelos de esta herramienta la cual identifica de forma más acertada el declive en $50 \%$ de la función renal tanto en filtrado glomerular o enfermedad renal en etapa terminal en muestras identificadas con nefropatía IgA en poblaciones asiáticas y europeas. Uno de estos modelos incluye el factor de etnicidad para catalogar de forma efectiva a estas razas, en el otro no se toma en cuenta y está destinada a aquellas poblaciones que no forman parte de las establecidas. Esta última es la herramienta de predicción que se utiliza para la población mexicana, ya que, de lo contrario, se nos estarían aplicando normas no aplicables a nuestra etnicidad, lo cual pudiera modificar los resultados.

Esta herramienta es un gran avance para la correcta estadificación de pacientes que corren alto riesgo de ser progresiva, incrementando la especificidad de futuros estudios y tratamientos. Además de que es más fácil de implementar ya que la mayoría de las variables que utiliza son pedidos rutinariamente durante las biopsias renales. Algunos de los elementos que incluye esta herramienta son la tasa de filtrado glomerular, la presión arterial, nivel de proteinuria, edad al momento de la biopsia, la raza del paciente, el uso de Inhibidores ACE o $A R B$ (bloqueador del receptor de angiotensina), el uso de inmunosupresores y el score MEST que ya ha sido validado internacionalmente. Es importante resaltar que la clasificación MEST-C sólo es aplicable a aquellos sujetos que aún no son tratados con inmunosupresión, puesto que anteriormente esta observación histológica se realizaba a todos los pacientes.

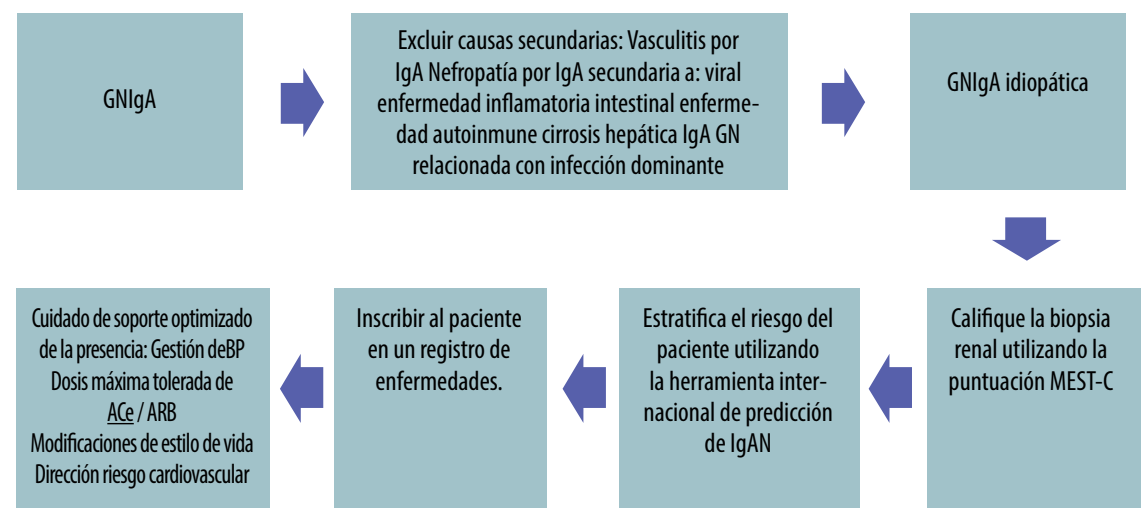

Figura 1. Evaluación inicial y manejo del paciente con GNIgA del KDIGO 2020 traducida al español. 
Para el paciente del caso expuesto, los valores que constituyen elementos de la herramienta de predicción de acuerdo con la guía KDIGO 2020 y la clasificación MEST-C, son los contenidos en la tabla 4. Se hace mención que el paciente del caso clínico tiene un puntaje MEST-C de CO, ya que éste es un criterio que no se incluye en "The International IgAN Prediction Tool"; todos los demás valores de la clasificación del Oxford vienen dentro de los criterios manejados por esta herramienta. El riesgo de una disminución del $50 \%$ en la TFG estimada o progresión a enfermedad renal en etapa terminal en 0.5 años después de la biopsia renal es $1.56 \%$, al año es de $6.19 \%$ y a los 5 años es de $59.26 \% .^{20}$

\section{Tabla 4}

"The International IgAN Prediction Tool" con los valores del sujeto de estudio.

\begin{tabular}{|l|c|}
\hline Tasa de filtrado glomerular & $59.4 \mathrm{ml} / \mathrm{min} / 1.73 \mathrm{~m} 2$ \\
\hline Presión sistólica durante la toma de biopsia & $145 \mathrm{mmHg}$ \\
\hline Presión diastólica durante la toma de biopsia & $90 \mathrm{mmHg}$ \\
\hline Nivel de proteinuria durante biopsia & $4.66 \mathrm{~g} / \mathrm{día}$ \\
\hline Edad & 24 años \\
\hline Raza & Otro (Hispano) \\
\hline Uso de inhibidores ACE o ARB al momento de la biopsia & Sí \\
\hline MEST M & 1 \\
\hline MEST E & 0 \\
\hline MEST S & 1 \\
\hline MEST T & 1 \\
\hline Uso de inmunosupresión durante o antes de la biopsia & No \\
\hline ¿A los cuántos meses después de la biopsia renal le gustaría & $6 / 12 / 60$ meses \\
\hline determinar el riesgo de progresión de daño renal?
\end{tabular}

Si no se dispone de esta clasificación, se puede decidir iniciar tratamiento de inmunosupresión en pacientes con GNIgA tomando en cuenta los siguientes puntos: 1) disminución progresiva de la TFG, 2) biopsia renal con datos de enfermedad activa, manifestado por grandes depósitos de IgA, y 3) proteinuria superior a $1 \mathrm{gr} /$ día persistente a pesar de tratamiento. El punto de mayor peso para la decisión de iniciar inmunosupresión es la proteinuria. ${ }^{15}$

El tratamiento de inmunosupresión en nuestro paciente fue iniciado con prednisona, ya que en varios estudios se ha com- probado que este medicamento disminuye el grado de proteinuria y la progresión del daño renal. ${ }^{15}$ El beneficio de la terapia demostró ser mayor en los casos que se han utilizado dosis más altas de glucocorticoides y cuando el tratamiento duró menos de 12 meses.

Dentro de los lineamientos de uso de glucocorticoides de acuerdo con las directrices KDIGO de 2020, se eligió prednisona de $1 \mathrm{mg} / \mathrm{kg} /$ día, máximo $75 \mathrm{mg} /$ día con duración de 2 meses, seguido de reducciones de dosis mensuales de $0.2 \mathrm{mg} / \mathrm{kg}$ durante un periodo de 6 meses (este es- 
quema es utilizado en el estudio de Manno C.). Es recomendable acompañar el uso de esteroides junto con profilaxis contra pneumocystis, protección gástrica y ósea. ${ }^{15} \mathrm{La}$ situación especial con el paciente reportado en esta revisión es la presencia de hipertensión, obesidad y dislipidemia, que se encontraban presentes desde el inicio del cuadro clínico y que pueden ser agravados con el uso de prednisona. Por lo cual se decide iniciar dosis de prednisona a $0.5 \mathrm{mg} / \mathrm{kg}$ (50 $\mathrm{mg}$ /día) por 3 meses, dosis con efecto sistémico moderado buscando la reducción del uso del esteroide dentro de 6 meses.

El enfoque principal del tratamiento de pacientes con GNIgA siempre serán las medidas de apoyo, vigilando el riesgo cardiovascular del paciente (disminución de la ingesta de sodio, dejar el tabaco, control del peso y la realización de actividad física). Los objetivos terapéuticos son múltiples para el paciente, incluyendo a) control hipertensivo, b) control del grado de proteinuria, llevándose a cabo con medicamentos que disminuyan la presión intraglomerular, y c) acompañado de manejo nutricional de la obesidad y dislipidemia con un menor aporte lipídico y una disminución del aporte proteico de origen animal, puesto que genera efectos hemodinámicos sobre la vasculatura intrarrenal como la vasodilatación de la arteriola eferente. 9,20 Éstos son los pilares fundamentales para disminuir el grado de proteinuria, y sólo de esta manera que lograremos preservar y promover un mejor funcionamiento renal.

\section{Conclusiones}

El presente caso clínico, explora la presentación laboratorial de GNIgA idiopática de una manera poco frecuente. La ausencia de hematuria es tal vez el principal hallazgo en la presentación del caso, ya que sin su presencia es difícil la sospecha clínica de GNIgA, dato importante al tratarse de la glomerulonefritis primaria más frecuente en el mundo. La aparición de síndrome nefrótico y la presencia de proliferación mesangial establecen la relación fisiopatológica entre estos hallazgos y debe hacerse hincapié en que los hallazgos obtenidos en la biopsia renal tengan su correlación clínica para poder asociar diagnóstico histopatológico y clínico.

La revisión del caso permite valorar las directrices actuales para el protocolo de estudio de los pacientes con GNIgA y poder hacer uso de las herramientas para la decisión final de iniciar manejo inmunosupresor y sobre todo generar conocimiento sobre el pronóstico del paciente, permitiendo al médico el ajuste de manejo de manera temprana y el poder ofrecer a los pacientes un pronóstico objetivo en términos de función renal, que es la principal preocupación de los pacientes al acudir a consulta.

Para este paciente el objetivo de manejo médico será evitar la hiperfiltración glomerular y frenar la proteinuria para evitar la esclerosis glomerular y fibrosis intersticial. Estos últimos hallazgos no fueron reportados de manera inicial en la biopsia, motivo del inicio de esteroide durante 6 meses tratando de mejorar la proteinuria y evitar la aparición de estas lesiones. Un reto importante en este paciente es el hallazgo de dislipidemia y obesidad grado 3, lo cual limita la dosis de esteroide y su uso a largo plazo, hallazgos que en nuestro medio es constante por la alta frecuencia de obesidad. Deberá buscarse el menor efecto secundario endocrinológico y vascular 
en estos pacientes, estableciendo el uso de manejo conjunto nutricional como parte fundamental en estos pacientes.

Como en la mayoría de las glomerulonefritis, no existe un adecuado registro en México ni en América Latina, siendo este tipo de artículos de reporte de caso clínico la mejor forma de mantener información actualizada sobre presentación de enfermedades de manera comunitaria.

\section{Bibliografía}

1. Steddon S, Chesser A, Cunningham Jet, et al. Oxford Handbook of Clinical Nephrology and Hypertension. 2nd. Ed,. New York. Oxford University Press; 2015

2. Benítez Llanes O, Henrry Pavier J, Castañer Moreno J, Fuentes Abreu J. Elementos de predicción en la nefropatía por inmunoglobulina A. Rev Cubana Med [Internet]. 1996 Abr [citado 2020 Jun 07];35(1):9-16. Disponible en: http://scielo.sld. $\mathrm{cu} /$ scielo.php? script $=$ sci_arttext $\&$ pid $=$ S003475231996000100003\&lng =es.

3. Hall $Y$, Fuentes E, Chertow G. Race/ethnicity and disease severity in IgA nephropathy. BMC Nephrol 5. [Internet]. 02 September 2004 [citado 6 Mayo del 2020]. Disponible en: https://doi. org/10.1186/1471-2369-5-10 (V)

4. Arias L. F, Henao J, Giraldo R. D, Carvajal N, Rodelo J, Arbeláez M. Glomerular diseases in a Hispanic population: review of a regional renal biopsy database. Sao Paulo Med. J. [Internet]. 2009 [citado 07 junio 2020] ; 127( 3 ): 140-144. Disponible en : http:// www.scielo.br/scielo.php?script=sci_arttext\&pid $=S$ $151631802009000300006 \&$ lng $=$ en. http://dx.doi. org/10.1590/S1516-31802009000300006.

5. O'Shaughnessy M. M, Hogan S. L, Thompson B. D Coppo, R, Fogo A. B, Jennette J. C, Glomerular disease frequencies by race, sex and region: results from the International Kidney Biopsy Survey. Nephrology, dialysis, transplantation : official publication of the European Dialysis and Transplant Association - European Renal Association [Internet] 2018 [citado 7 junio 2020]; 33(4), 661-669. Disponible en: https:// doi.org/10.1093/ndt/gf×189

6. Ortega-Alonzo SE, Hinojosa-Lezama JM. Hematuria macroscópica: a propósito de un caso con sospecha de enfermedad de Berger. Rev Mex Pediatr. [Internet] 2016 [citado 12 junio 2020]; 83(6): 203-207. Disponible en: https://www.medigraphic.com/pdfs/ pediat/sp-2016/sp166f.pdf

7. Valencia V. C, de La Cruz C. O, Fuentes J. G. B, Ramírez F. F, Michel R. P, Aragaki, Y, Cárdenas M. A. G, Epidemiología de la enfermedad glomerular en adultos. Revisión de una base de datos. Gac Med Mex [Internet] 2014 [citado 18 junio 2020]; 150(5), 403408. Disponible en: https://www.medigraphic.com/ pdfs/gaceta/gm-2014/gm145c.pdf

8. Durán ÁS, Campo SL, La nefropatía IgA, la glomerulopatía más frecuente en el mundo. Rev Cubana
Pediatr. [Internet] 2015 [citado 15 Junio 2020]; 87(3):350-361. Disponible en: https://www.medigraphic.com/pdfs/revcubped/cup-2015/cup153j. pdf

9. Cheung C. K, Barratt J, Fervenza F. C. Clinical presentation and diagnosis of IgA nephropathy. UpTo Date [Internet] 2020 [citado 15/06/2020] Disponible en: https://www-uptodate-com.dibpxy.uaa. $\mathrm{mx} /$ contents/clinical-presentation-and-diagnosisof-iga-nephropathy? search =Clinical\% 20presentation $\% 20$ and $\%$ 20diagnosis $\% 20$ of\% 20lgA $\% 20$ nephropathy \&source $=$ search_result\&selectedTitle $=$ $1 \sim 121$ \&usage type $=$ default $\$$ display $r a n k=1$

10. Valencia V. C, de La Cruz C. O, Fuentes J. G. B, Ramírez F. F, Michel R. P, Aragaki Y, Cárdenas, M. A. G, Epidemiología de la enfermedad glomerular en adultos. Revisión de una base de datos. Gaceta médica de México [Internet] 2014 [ citado 24 Junio 2020]; 150(5), 403-408. Disponible en: https://www.medigraphic.com/pdfs/gaceta/gm-2014/gm145c.pdf

11. Cattran D.C. y Appel G.B., Treatment and prognosis of IgA nephropathy. UpToDate. [Internet] 2020 [citado 15/06/2020] Disponible en: https://www-uptodatecom.dibpxy.uaa.mx/contents/treatment-and-prognosis-of-iga-nephropathy?search $=$ tratamiento $\% 20$ de $\% 20$ nefropat $\%$ C $3 \%$ A D a $\% 20$ por $\% 20$ IgA\&source $=$ search_result\&selectedTitle $=1 \sim 121 \&$ usage_type $=$ default $\&$ display_rank=1\# $\mathrm{H} 13$

12. Barbour SJ, Coppo R, Zhang $\mathrm{H}$, et al. Evaluating a New International Risk-Prediction Tool in IgA Nephropathy. JAMA Intern Med. [ internet] 2019; [ consultado 28/12/2020 ] 179(7):942-952. doi:10.1001/jamainternmed.2019.0600

13. Choung, Hae-Yoon G, et al. The spectrum of kidney biopsy findings in patients with morbid obesity. Kidney International [Internet] 2018 [ Citado 15 de Junio de 2020] Volume 95, Issue 3, 647 - 654. Disponible en: https://www.kidney-international.org/ article/S0085-2538(18)30907-4/fulltext

14. Navarro G, Ardiles L, Obesidad y enfermedad renal crónica: Una peligrosa asociación. Rev. méd. Chile [Internet] Enero 2015 [citado 15 Junio 2020] ; 143( 1 ): 77-84. Disponible en: https://scielo.conicyt.cl/scielo.php?script $=$ sci arttext $\&$ pid $=$ S0034 98872015000100010\&lng=es. $\quad$ http://dx.doi. org/10.4067/S0034-98872015000100010

15. Lococo B, Alberton V, Fazzini B, Smuclir Quevedo A, Morales D, Malvar A, Nefropatía por IgA. Revisión y conducta terapéutica a propósito de un caso clínico. 
Revista de Nefrología, Diálisis y Trasplante. [Internet] 2016 [Citado 8 Mayo 2020]; 36(2), 108-123. Disponible en: https://168.197.50.143/index.php/ rndt/article/view/66

16. Rivera Gorrin, M., \& Quereda Rodríguez-Navarro, C. La ecografía realizada por el nefrólogo: nuestra experiencia. Nefrología (English Edition). [Internet] 2009 [Consultado el 15 de diciembre del 2020] 2(1), 9-16. Disponible en https://www.revistanefrologia.com/es-relaccionados-la-ecografia-realizada-por-el-nefrologo-nuestra-experiencia-articulo-X1888970009000363

17. Cattran DC, Greenwood C, Ritchie S. Long-term benefits of angiotensin-converting enzyme inhibitor therapy in patients with severe immunoglobulin a nephropathy: a comparison to patients receiving treatment with other antihypertensive agents and to patients receiving no therapy. Am J Kidney Dis [Internet] 1994 [Citado 14/06/2020]; 23(2) pag. 247-254. Disponible en: https://www.sciencedirect.
com/science/article/abs/pii/S0272638612809802

18. Kanno Y, Okada H, Yamaji Y, Nakazato $Y$, Suzuki $H$, Angiotensin-converting-enzyme inhibitors slow renal decline in IgA nephropathy, independent of tubulointerstitial fibrosis at presentation, QJM [Internet] 2005 [Citado 14/06/2020]; 98(3), Pag. 199-203. Disponible en: https://doi.org/10.1093/qjmed/ hci036

19. Li P. K. T, Kwan B. C. H, Chow K. M, Leung C. B, Szeto $C$. C, Treatment of early immunoglobulin A nephropathy by angiotensin-converting enzyme inhibitor. Am J Med [Internet] 2013 [Citado 13/06/2020]; 126(2), Pag. 162-168. Disponible en: https://doi. org/10.1016/j.amjmed.2012.06.028

20. International IgAN Prediction Tool - Adults Calcutae [Internet]. (MX): QxMD; 2020 [Citado 12 de Julio 2020]. QxMD; [2] Disponible en: https://qxmd. com/calculate/calculator_499?_branch_match_ id $=656546875419766679$ 